\title{
Aberrant Left Hepatic Artery Arising from Left Gastric Artery at Curative Gastrectomy for Gastric Cancer
}

\author{
Chansik An, M.D., Joon-Seok Lim, M.D. \\ Department of Radiology, Research Institute of Radiological Science, Severance Hospital, Yonsei University College of Medicine, \\ Seoul, Korea
}

\begin{abstract}
An aberrant left hepatic artery is one of the most common variants of hepatic arteries, and its prevalence has been reported 6.5-30\%. During D2 lymph node dissection for gastric cancer, an aberrant left hepatic artery arising from left gastric artery is ligated which may lead to hepatic damage. In this case report, a 66-year-old male patient underwent total gastrectomy with D2 lymph node dissection during which the aberrant left hepatic artery was ligated. Post-operative liver function tests revealed elevated liver enzymes, and ischemic changes in the left lateral hepatic section was seen on the CT scan. On retrospective review of preoperative CT images, a replaced left hepatic artery from left gastric artery could have been identified. The information on the presence of aberrant LHA and its supplying area is clinically important when planning curative gastrectomy for gastric cancer, because extended lymph node dissection requires division of the left gastric artery and this may lead to severe liver damage. By using preoperative CT scan, detection of aberrant left hepatic artery could be done.
\end{abstract}

Key Words Stomach cancer · CT · Aberrant left hepatic artery.

Received: November 17, 2014 / Revised: November 18, 2014 / Accepted: December 1, 2014

Address for correspondence: Joon-Seok Lim, M.D.

Department of Radiology, Severance Hospital, 50 Yonsei-ro, Seodaemun-gu, Seoul 120-752, Korea

Tel: 82-2-2228-7400, Fax: 82-2-393-3035, E-mail: jslim1@yuhs.ac

\section{Introduction}

Surgery is currently the only potentially curative treatment for gastric cancer. For standard curative surgery, the complete resection and extended lymph node dissection (D2) are performed as it improves long-term survival without increases in morbidity or mortality in patients with stage II, IIIA, and perhaps IIIB disease (1). In the presence of an aberrant left hepatic artery (LHA) arising from left gastric artery (LGA), the aberrant LHA is ligated during D2 lymph node dissection which may lead to hepatic damage. We report a case where ligation of an aberrant LHA led to ischemic insult to left lateral hepatic section, and discuss the importance of preoperative assessment of hepatic vasculature.

\section{Case Report}

A 66-year-old male patient visited our hospital with a chief complaint of epigastric pain for one month. Endoscopic examination revealed an ulceroinfiltrative lesion with an irregular base and a nodular elevated margin on the greater curvature of the low to middle body of the stomach (Fig. 1). The histologic diagnosis from the biopsy samples was adenocarcinoma. Preoperative liver function tests were within normal limits, with an aspartate transaminase (AST) level of $20 \mathrm{IU} / \mathrm{L}$ (normal= 5-41 $\mathrm{IU} / \mathrm{L}$ ) and an alanine transaminase (ALT) level of $27 \mathrm{IU} / \mathrm{L}$ (normal=17-59 IU/L). The serological workups for the hepatitis $B$ virus antigen and hepatitis $C$ virus antibody were negative. A radical surgery was then planned, and routine total gastrectomy with extended lymph node dissection was performed. Pathologic staging according to the TNM classification was T2N2M0 (stage IIB), 9 out of 63 lymph nodes removed at surgery were positive for metastasis (station 1 and 3). Liver function tests taken immediately after the surgery revealed elevated serum aminotranferases, with an AST level of $102 \mathrm{IU} / \mathrm{L}$ and an ALT level of 116 IU/L. In subsequent liver function tests, AST/ALT elevated up 

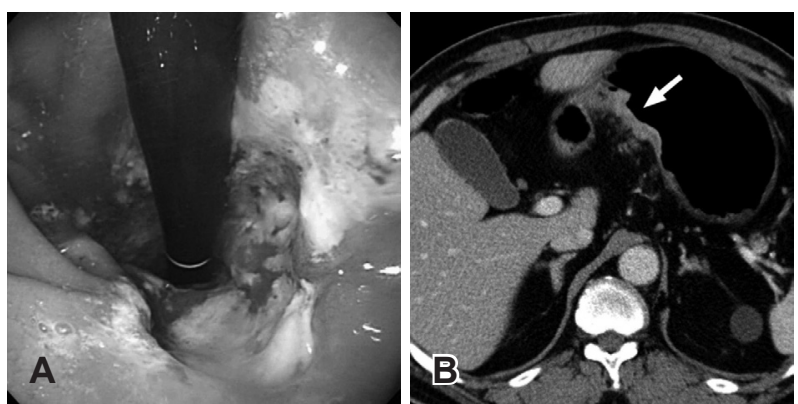

Fig. 1. A 66-year-old male underwent EGD and CT scan. Advanced gastric cancer was found on the greater curvature of the low to middle body of the stomach on EGD (A) and CT scan (B, arrow).

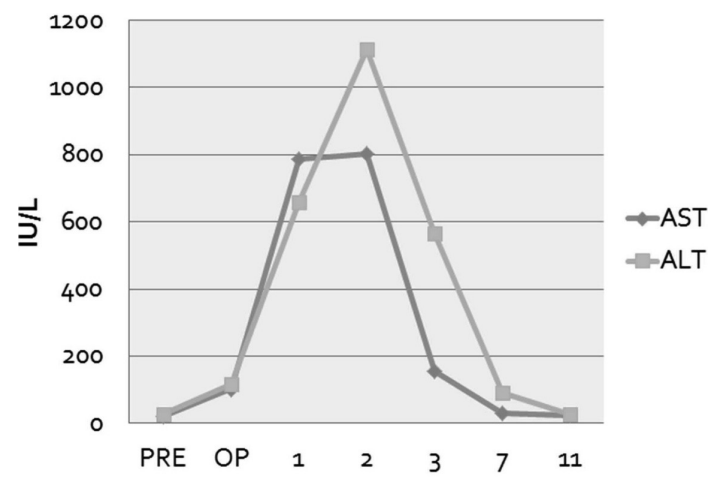

Fig. 2. Serial Changes in Postoperative Liver Function.
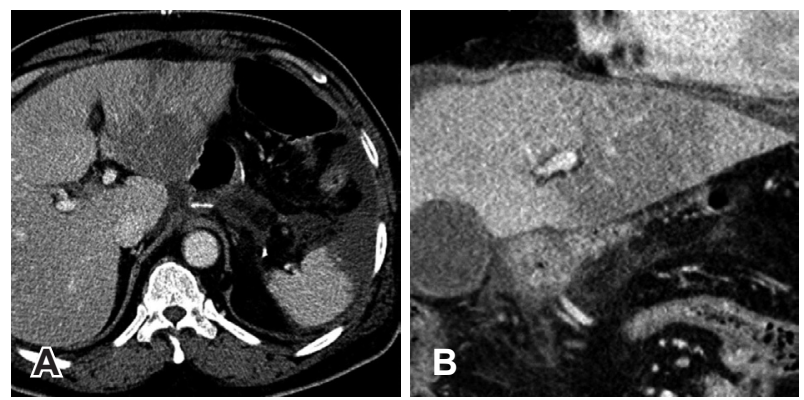

Fig. 3. Contrast-enhanced CT scan taken two days after the surgery. A hypoattenuating geographical area due to ischemic insult is found in the left lateral section of the liver (A: transverse image, B: coronal image).
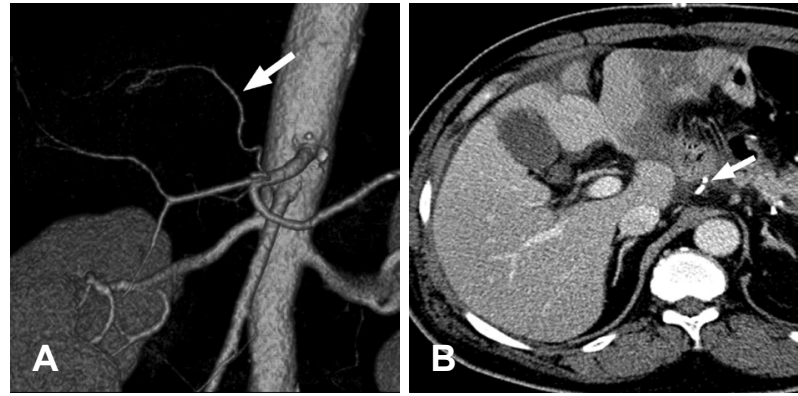

Fig. 4. Comparison of the preoperative and postoperative CT scans. A: Volume-rendered image of the preoperative CT scan shows an aberrant left hepatic artery (arrow) from the left gastric artery. B: Transverse image of the postoperative CT scan shows a surgical clip used to ligate the root of the left gastric artery (arrow). to $803 / 1,115 \mathrm{IU} / \mathrm{L}$ until normalized by the 11th post-operative day. Fig. 2 shows serial changes in postoperative liver function. Contrast-enhanced CT scan taken two days after the surgery showed a geographical area of decreased perfusion in the left lateral section of the liver (Fig. 3). Comparing the preoperative and postoperative CT scans, this patient had a replaced LHA arising from LGA, and the root of LGA was ligated at gastrectomy with lymph node dissection (Fig. 4). These findings suggest that the decreased perfusion in the left lateral section found on the CT scan after the surgery is due to ischemia caused by ligation of the replaced LHA. The patient was discharged in the third postoperative week without other complications.

\section{Discussion}

In the standard, or classic, vascular anatomy, the celiac artery gives rise to three branches: LGA, common hepatic artery (CHA), and splenic artery. The proper hepatic artery arises from the $\mathrm{CHA}$, which in turn bifurcates into right and left hepatic arteries to supply the entire liver. This standard hepatic arterial anatomy has been reported in approximately $50 \%$ of patients on the basis of cadaveric and early angiographic reports (2). Aberrant (non-standard) hepatic arteries can have two types, replaced and accessory. An aberrant replaced hepatic artery is a substitute for the standard hepatic artery that is absent. An aberrant accessory hepatic artery appears in addition to one that is normally present.

One of the most common variants of hepatic arteries is an aberrant (including both replaced and accessory) LHA from LGA, which has been reported in $6.5-30 \%$ in the literature $(2,3)$. This aberrant LHA has a clinical significance in surgery of the upper intestinal tract, because extended lymph node dissection requires division of the LGA during curative gastrectomy for gastric cancer. Lymph node metastasis of gastric cancer is most frequently encountered around the LGA, and complete dissection of this area is an important element of curative surgical treatment for gastric cancer $(4,5)$. In the presence of an aberrant LHA from LGA, however, this procedure requires ligation and division of the aberrant LHA, which may lead to liver dysfunction, as seen in this case.

However, no consensus exists regarding the indications for preservation or ligation of replaced LHA, although there would not be much argument about ligation of accessory LHA. Shinohara et al found no significant differences in the total number of lymph nodes and metastatic lymph nodes around the LGA, morbidity, mortality, and overall 5-year survival between patients whose aberrant LHAs were preserved or ligated (3). Therefore, they reported that preserving aberrant LHA may be able to avoid 
postoperative liver damage and routine division of the aberrant LHA, particularly replaced LHA does not be required as long as it is not directly involved by the tumor.

By reviewing the surgical records of a group of 141 patients who had undergone gastrectomy for gastric cancer following preoperative angiography, Okano et al found that the wider the area fed by the LHA from LGA, the greater was the change in liver function, and they concluded that ligation of a thin accessory hepatic artery does not inevitably result in severe liver damage (6).

Therefore, the identification of aberrant LHA is important before gastric surgery. Recently CT, and in particular, multidetector-row CT, has superseded conventional angiography in the study of the hepatic arteries in addition to its role in preoperative staging, as several studies showed an excellent correlation between angiography and CT angiography in hepatic artery evaluation (7).

\section{Conclusion}

The information on the presence of aberrant LHA and its supplying area is clinically important when planning curative gastrectomy for gastric cancer, because extended lymph node dissection requires division of the LGA and this may lead to severe liver damage. By using preoperative CT scan, detection of aber- rant LHA and differentiation of replaced LHA and accessory LHA could be done.

\section{REFERENCES}

1. Dicken BJ, Bigam DL, Cass C, Mackey JR, Joy AA, Hamilton SM. Gastric adenocarcinoma: Review and considerations for future directions. Annals of Surgery 2005;241:27-39

2. Covey AM, Brody LA, Maluccio MA, Getrajdman GI, Brown KT. Variant hepatic arterial anatomy revisited: Digital subtraction angiography performed in 600 patients. Radiology 2002;224:542-547

3. Shinohara T, Ohyama S, Muto T, Yanaga K, Yamaguchi T. The significance of the aberrant left hepatic artery arising from the left gastric artery at curative gastrectomy for gastric cancer. European journal of surgical oncology: the journal of the European Society of Surgical Oncology and the British Association of Surgical Oncology 2007;33: 967-971

4. Maruyama K, Gunven P, Okabayashi K, Sasako M, Kinoshita T. Lymph node metastases of gastric cancer. General pattern in 1931 patients. Annals of surgery 1989;210:596-602

5. Sasako M, McCulloch P, Kinoshita T, Maruyama K. New method to evaluate the therapeutic value of lymph node dissection for gastric cancer. The British Journal of Surgery 1995;82:346-351

6. Okano S, Sawai K, Taniguchi H, Takahashi T. Aberrant left hepatic artery arising from the left gastric artery and liver function after radical gastrectomy for gastric cancer. World Journal of Surgery 1993;17: 70-73; discussion 74

7. Saba L, Mallarini G. Multidetector row ct angiography in the evaluation of the hepatic artery and its anatomical variants. Clinical Radiology 2008;63:312-321 